\title{
Hydrodynamic correlations in multiparticle collision dynamics fluids
}

\author{
Chien-Cheng Huang, Gerhard Gompper, and Roland G. Winkler \\ Theoretical Soft Matter and Biophysics, Institute of Complex Systems, Institute for Advanced Simulation, \\ Forschungszentrum Jülich, D-52425 Jülich, Germany \\ (Received 28 August 2012; published 27 November 2012)
}

\begin{abstract}
The emergent fluctuating hydrodynamics of the multiparticle collision dynamics (MPC) approach, a particlebased mesoscale simulation technique for fluid dynamics, is analyzed theoretically and numerically. We focus on the stochastic rotation dynamics implementation of the MPC method. The fluid is characterized by its longitudinal and transverse velocity correlation functions in Fourier space and velocity autocorrelation functions in real space. Particular attention is paid to the role of sound, which leads to piecewise negative correlation functions. Moreover, finite system-size effects are addressed with an emphasis on the role of sound. Analytical expressions are provided for the transverse and longitudinal velocity correlations, which are derived from the linearized Landau-Lifshitz Navier-Stokes equation adopted for an isothermal MPC fluid. The comparison of the analytical results with simulations shows excellent agreement above a minimal length scale. The simulations indicate a breakdown in hydrodynamics on length scales smaller than this minimal length. This demonstrates that we have an excellent analytical description and understanding of the MPC method and its limitations in terms of time and length scales.
\end{abstract}

DOI: 10.1103/PhysRevE.86.056711

PACS number(s): 47.11.-j, 47.15.G-, 05.40.-a, 02.70.Ns

\section{INTRODUCTION}

The desire to bridge the disparate time, length, and energy scales of soft matter and biological systems has stimulated the development of various mesoscale simulation techniques. A particularly appealing method is the multiparticle collision dynamics (MPC) approach proposed by Malevanets and Kapral [1,2]. MPC is a particle-based simulation technique, which incorporates thermal fluctuations, provides hydrodynamic correlations, and is easily coupled with other simulation techniques, such as molecular dynamics simulations for embedded particles [3,4]. MPC proceeds in two steps-a streaming and a collision step. Collisions occur at fixed discrete time intervals, and although space is discretized into cells to define the multiparticle collision environment, both particle coordinates and velocities are continuous variables. Various schemes for the collision interaction have been proposed [1-7]. The original method, which employs rotation of relative velocities, is often denoted as stochastic rotation dynamics (SRD) [1-6].

The MPC approach has successfully been applied to a broad range of soft matter systems, such as colloids $[1,3,4,8-15]$ and polymers [3,4,16-19] under equilibrium conditions. Similar nonequilibrium properties have been studied for colloids [7,20-26], polymers [19,27-34], vesicles [35], and cells $[36,37]$ in flow fields, colloids in viscoelastic fluids [38], as well as for self-propelled spheres [39-41], rods [3,42], and other swimming objects [43-45]. Moreover, extensions have been proposed for fluids with nonideal equations of state [46] and mixtures [47]. The simulation of such systems is often rather demanding in terms of computational resources since it can involve as much as $10^{4}-10^{6}$ embedded particles and $10^{6}-10^{9}$ MPC particles. Hence, such systems can only be studied on massively parallel platforms $[19,48]$.

In this article, we discuss the hydrodynamic properties of the SRD version of a MPC solvent. For an analytical description of the fluid, we adopt a fluctuating hydrodynamic approach based on the linearized Landau-Lifshitz
Navier-Stokes equation, i.e., thermal fluctuations are taken into account. Since the stress tensor of the MPC fluid is nonsymmetric [11,49-53], adjustments of the fluctuating stress tensor are necessary. By comparing theoretical with simulation results, we want to achieve a detailed understanding of the hydrodynamic behavior of the fluid on a broad range of length and time scales. As is well known, in MPC, hydrodynamics certainly breaks down on the length scale of a collision cell. However, less evident is how the smallest hydrodynamic length scale depends on the interval between MPC collisions.

Another aspect is the compressibility of the MPC fluid. We want to characterize the influence of sound on the longitudinal current correlations and the MPC-particle velocity autocorrelation function. For micrometer-size colloids in water, there is a clear separation of the sonic time scale $\tau_{c}$ from the viscous time scale $\tau_{v}$; since $\tau_{c} \ll \tau_{v}$, sound effects can be neglected. However, recent theoretical [54] and simulation papers [15] reveal that sonic and viscous effects interfere and give rise to an effect denoted as backtracking. Then, the fluid or embedded particle velocity correlation functions no longer decay monotonically but may even become negative, exhibiting viscoelasticlike behavior. Such effects appear, specifically for compressible fluids, when the sonic time scale becomes comparable to the viscous time scale. Indeed, experiments on colloidal systems revealed fluid-induced correlations on time scales $t<\tau_{v}$ [55,56], which are attributed to sonic effects.

A quantitative characterization of a fluid and, in particular, the MPC fluid, is useful in several respects. A particular example is the appearance of screening of hydrodynamic interactions in semidilute polymer solutions [57]. Understanding of the fluid behavior is a prerequisite for interpreting the complex polymer dynamics, which is intimately coupled to the fluid. In that respect, by its particle nature, MPC provides access to both the polymer and the fluid dynamics.

The paper is organized as follows: In Sec. II, the MPC method is briefly described, and adopted parameters are 
stated. The fluctuating hydrodynamic approach is outlined in Sec. III. Longitudinal and transverse velocity correlation functions are calculated, in particular, for a periodic system. MPC fluid velocity correlation functions are presented and are compared with theoretical predictions in Sec. IV. Finally, Sec. V summarizes our findings.

\section{MODEL}

In MPC [1-4], the solvent is modeled by point particles, which move ballistically (streaming) between local multiparticle collisions, an imposed stochastic process. We consider a system of $N$ particles of mass $m$ in a periodic system of volume $V=L^{3}$. During the streaming step, the particles change their positions $\boldsymbol{r}_{i}(t)$ according to

$$
\boldsymbol{r}_{i}(t+h)=\boldsymbol{r}_{i}(t)+h \boldsymbol{v}_{i}(t)
$$

where $t$ is the time, $h$ is the collision time, $\boldsymbol{v}_{i}(t)$ is the velocity of particle $i$, and $i=1, \ldots, N$. For the collisional interaction, the simulation box is divided into cubic collision cells of side length $a$. In the SRD version of MPC, the relative velocity of each particle, with respect to the center-of-mass velocity of the cell, is rotated by a fixed angle $\alpha$ around a randomly oriented axis,

$$
\boldsymbol{v}_{i}(t+h)=\boldsymbol{v}_{i}(t)+[\mathbf{R}(\alpha)-\mathbf{E}]\left[\boldsymbol{v}_{i}(t)-\boldsymbol{v}_{\mathrm{cm}}(t)\right] .
$$

$\mathbf{R}(\alpha)$ is the rotation matrix, $\mathbf{E}$ is the unit matrix, and

$$
\boldsymbol{v}_{\mathrm{cm}}=\frac{1}{N_{c}} \sum_{j=1}^{N_{c}} \boldsymbol{v}_{j}
$$

is the center-of-mass velocity of the particles in the cell of particle $i$, and $N_{c}$ is the total number of solvent particles in that cell [1-4]. In its original versions, MPC breaks Galilean invariance $[1,2,5]$. To ensure Galilean invariance, a random shift is performed at every collision step [5]. In a collision step, mass, momentum, and energy are conserved, which leads to the buildup of correlations between the particles and gives rise to hydrodynamic interactions.

We consider isothermal rather than isoenergetic systems because we are typically interested in nonequilibrium systems where temperature has to be controlled. A constant temperature is achieved by a local Maxwellian thermostat where we scale the relative velocities within a collision cell according to the Maxwell-Boltzmann scaling (MBS) method [58]. In the MBS method, an energy value is taken from the known distribution function ( $\Gamma$ distribution) of the kinetic energy of a collision cell. The ratio of this energy and the actual kinetic energy determines the scale factor. This procedure yields a local Maxwellian distribution of relative velocities both in equilibrium and in nonequilibrium simulations [58].

The simulations are performed with the rotation angle $\alpha=130^{\circ}$ and mean number of particles $\left\langle N_{c}\right\rangle=10$ per collision cell. The collision times $h / \sqrt{m a^{2} /\left(k_{B} T\right)}=1,0.5$, $0.1,0.05,0.02$, and 0.01 are used, which correspond to the viscosities $\eta / \sqrt{m k_{B} T / a^{4}}=5.78,4.07,8.7,16.7,41.2$, and 82.2 , respectively. The length of the simulation box is $L=60 a$ if not otherwise stated.

\section{FLUCTUATING HYDRODYNAMICS}

As shown in Refs. [1-3,49-51], the hydrodynamic properties of the MPC fluid can be described by the linearized Navier-Stokes equations on sufficiently large length and time scales. For an isothermal fluid system, mass and momentum conservation are expressed by the continuity equation and the Navier-Stokes equations,

$$
\begin{gathered}
\frac{\partial}{\partial t} \rho+\nabla \cdot(\rho \boldsymbol{v})=0 \\
\rho\left[\frac{\partial}{\partial t} \boldsymbol{v}+(\boldsymbol{v} \cdot \nabla) \boldsymbol{v}\right]=\nabla \cdot \boldsymbol{\sigma}+\boldsymbol{f} .
\end{gathered}
$$

Here, $\rho=\rho(\boldsymbol{r}, t)$ denotes the mass density of the fluid, $\boldsymbol{v}=$ $\boldsymbol{v}(\boldsymbol{r}, t)$ denotes the fluid velocity field at position $\boldsymbol{r}$ in space at time $t, f$ is a volume force, which we set to zero in the following, and $\sigma$ is the stress tensor.

The viscous stress tensor of a MPC fluid in $d$ dimensions consists of a symmetric kinetic stress tensor $\sigma_{\alpha \beta}^{\mathrm{k}}$ and a nonsymmetric collisional stress tensor $\sigma_{\alpha \beta}^{\mathrm{c}}$ where

$$
\begin{gathered}
\sigma_{\alpha \beta}^{\mathrm{k}}=\eta^{\mathrm{k}}\left[\frac{\partial v_{\beta}}{\partial r_{\alpha}}+\frac{\partial v_{\alpha}}{\partial r_{\beta}}-\frac{2}{d} \delta_{\alpha \beta} \sum_{\alpha^{\prime}} \frac{\partial v_{\alpha^{\prime}}}{\partial r_{\alpha^{\prime}}}\right], \\
\sigma_{\alpha \beta}^{\mathrm{c}}=\eta^{\mathrm{c}} \frac{\partial v_{\alpha}}{\partial r_{\beta}},
\end{gathered}
$$

as discussed in Refs. [49,50,52] with the collisional $\left(\eta^{\mathrm{c}}\right)$ and kinetic $\left(\eta^{\mathrm{k}}\right)$ parts of the MPC fluid viscosity $\eta=\eta^{\mathrm{k}}+\eta^{\mathrm{c}}[3,4$, 59]. $\alpha, \beta, \alpha^{\prime}, \beta^{\prime} \in\{x, y, z\}$ denote the Cartesian directions. The total stress tensor can then be written as [50-52]

$$
\begin{aligned}
\sigma_{\alpha \beta}= & \left(\eta^{\mathrm{k}}+\frac{1}{2} \frac{d}{d-1} \eta^{\mathrm{c}}\right)\left[\frac{\partial v_{\beta}}{\partial r_{\alpha}}+\frac{\partial v_{\alpha}}{\partial r_{\beta}}-\frac{2}{d} \delta_{\alpha \beta} \sum_{\alpha^{\prime}} \frac{\partial v_{\alpha^{\prime}}}{\partial r_{\alpha^{\prime}}}\right] \\
& +\frac{1}{2} \frac{d-2}{d-1} \eta^{\mathrm{c}}\left[\frac{\partial v_{\alpha}}{\partial r_{\beta}}-\frac{\partial v_{\beta}}{\partial r_{\alpha}}\right]-p \delta_{\alpha \beta}+\sigma_{\alpha \beta}^{R},
\end{aligned}
$$

up to a tensor of vanishing divergence, which, consequently, does not appear in linearized Navier-Stokes equations. $p$ denotes the hydrostatic pressure, and $\sigma_{\alpha \beta}^{R}$ accounts for the thermal fluctuations in the fluid [60-62]. We would like to emphasize two points. First of all, the stress tensor (8) is symmetric in two dimensions. Second, we set the bulk viscosity to zero. Our simulations of three-dimensional systems yield very small and, hence, negligible values for the bulk viscosity. Similarly, in Ref. [51], a negligible bulk viscosity was found for two-dimensional systems. This is consistent with the general expectation that the bulk viscosity is zero for ideal monatomic gases $[63,64]$.

The stochastic process for $\sigma^{R}$ is assumed to be Gaussian and Markovian with the moments,

$$
\begin{aligned}
\left\langle\boldsymbol{\sigma}^{R}\right\rangle & =0, \\
\left\langle\sigma_{\alpha \beta}^{R}(\boldsymbol{r}, t) \sigma_{\alpha^{\prime} \beta^{\prime}}^{R}(\boldsymbol{r}, t)\right\rangle & =2 k_{B} T \eta_{\alpha \beta \alpha^{\prime} \beta^{\prime}} \delta\left(\boldsymbol{r}-\boldsymbol{r}^{\prime}\right) \delta\left(t-t^{\prime}\right),
\end{aligned}
$$

and

$$
\begin{aligned}
\eta_{\alpha \beta \alpha^{\prime} \beta^{\prime}}= & \eta \delta_{\alpha \beta^{\prime}} \delta_{\beta \alpha^{\prime}}+\frac{1}{d-1}\left[\eta+(d-2) \eta^{\mathrm{k}}\right] \delta_{\alpha \alpha^{\prime}} \delta_{\beta \beta^{\prime}} \\
& -\frac{1}{d-1}\left[\eta+\frac{d-2}{d} \eta^{\mathrm{k}}\right] \delta_{\alpha \beta} \delta_{\alpha^{\prime} \beta^{\prime}} .
\end{aligned}
$$


Since the stress tensor is nonsymmetric, $\sigma^{R}$ is also nonsymmetric. In Eq. (10), we, therefore, extend the correlation functions, corresponding to a symmetric stress tensor [60-62,65], to our nonsymmetric case. The correlation functions of Refs. [60-62] for angular momentum conserving fluids are recovered for $\eta^{\mathrm{c}}=0$ and $\eta=\eta^{\mathrm{k}}$. Moreover, these correlation functions are also recovered in $d=2$ without any further assumption.

With the stress tensors (6), (7), or (8), Eq. (5) turns into the linearized Landau-Lifshitz Navier-Stokes equation [60],

$$
\rho \frac{\partial}{\partial t} \boldsymbol{v}=-\nabla p+\eta \Delta \boldsymbol{v}+\left(\frac{d-2}{d}\right) \eta^{\mathrm{k}} \nabla(\boldsymbol{\nabla} \cdot \boldsymbol{v})+\boldsymbol{f}^{R},
$$

at low Reynolds numbers. For convenience, we introduce the random force $f^{R}(\boldsymbol{r}, t)=\nabla \cdot \sigma^{R}$. In $d=2$, Eq. (11) reduce to the well-known equation for an incompressible fluid [60]. Since $\nabla \cdot v$ may not be zero, this, however, does not necessarily correspond to an incompressible fluid. In three dimensions, the nonsymmetric stress tensor leads to a term, which contributes to sound propagation and solely depends on the kinetic viscosity.

In the following, we will consider three-dimensional systems. Taking the divergence of Eq. (11), we arrive at the equation,

$$
\Delta p-\frac{1}{c^{2}} \frac{\partial^{2} p}{\partial t^{2}}=\nabla \cdot\left(\eta \Delta \boldsymbol{v}+\frac{\eta^{\mathrm{k}}}{3} \nabla(\nabla \cdot \boldsymbol{v})+\boldsymbol{f}^{R}\right),
$$

within linearized hydrodynamics. The second derivative with respect to time on the left-hand side follows from Eq. (4) together with the ideal gas equation of state, which applies to the MPC fluid. $c=\sqrt{k_{B} T / m}$ is the isothermal velocity of sound of the MPC fluid. To solve Eqs. (11) and (12), we perform Fourier transformations. Since we want to compare the analytical results with computer simulations, we adopt a discrete Fourier transformation in space, i.e., we use

$$
\begin{gathered}
\boldsymbol{v}(\boldsymbol{r}, t)=\frac{1}{2 \pi} \sum_{\boldsymbol{k}} \int \hat{\boldsymbol{v}}(\boldsymbol{k}, \omega) e^{-i \boldsymbol{k} \cdot \boldsymbol{r}} e^{i \omega t} d \omega, \\
\hat{\boldsymbol{v}}(\boldsymbol{k}, \omega)=\frac{1}{V} \int \boldsymbol{v}(\boldsymbol{r}, t) e^{i \boldsymbol{k} \cdot \boldsymbol{r}} e^{-i \omega t} d^{3} r d t,
\end{gathered}
$$

with $k_{\alpha}=2 \pi n_{\alpha} / L$ and $n_{\alpha} \in \mathbb{Z} \backslash\{0\}$. This yields

$$
\begin{array}{r}
i \omega \rho \hat{\boldsymbol{v}}=i \boldsymbol{k} \hat{\boldsymbol{p}}-\eta k^{2} \hat{\boldsymbol{v}}-\frac{\eta^{\mathrm{k}}}{3} k^{2} \mathbf{P} \hat{\boldsymbol{v}}+\hat{\boldsymbol{f}}^{R}, \\
\left(\frac{\omega^{2}}{c^{2}}-k^{2}\right) \hat{p}=i \boldsymbol{k} \cdot\left(\eta k^{2} \hat{\boldsymbol{v}}+\frac{\eta^{\mathrm{k}}}{3} k^{2} \mathbf{P} \hat{\boldsymbol{v}}-\hat{\boldsymbol{f}}^{R}\right),
\end{array}
$$

where $\mathbf{P}$ is a projection operator with the components $P_{\alpha \beta}=k_{\alpha} k_{\beta} / k^{2}$, which projects a vector along the direction of $\boldsymbol{k}$, and $k=|\boldsymbol{k}|$. With the splitting $\hat{\boldsymbol{v}}=\hat{\boldsymbol{v}}^{L}+\hat{\boldsymbol{v}}^{T}$ into a longitudinal $\hat{\boldsymbol{v}}^{L}$ and transverse part $\hat{\boldsymbol{v}}^{T}$ with respect to $\boldsymbol{k}$, i.e., $\hat{\boldsymbol{v}} \cdot \boldsymbol{k}=\hat{v}^{L} k$ and $\hat{\boldsymbol{v}}^{T} \cdot \boldsymbol{k}=0$, Eqs. (15) yield

$$
\hat{\boldsymbol{v}}(\boldsymbol{k}, \omega)=\left(\hat{\mathbf{Q}}^{L}+\hat{\mathbf{Q}}^{T}\right) \hat{\boldsymbol{f}}^{R},
$$

with

$$
\begin{aligned}
& \hat{\mathbf{Q}}^{L}=\left(\tilde{\eta} k^{2}+\frac{i \rho}{\omega}\left[\omega^{2}-c^{2} k^{2}\right]\right)^{-1} \mathbf{P}=\hat{Q}^{L} \mathbf{P}, \\
& \hat{\mathbf{Q}}^{T}=\left(\eta k^{2}+i \rho \omega\right)^{-1}(\mathbf{E}-\mathbf{P})=\hat{Q}^{T}(\mathbf{E}-\mathbf{P}),
\end{aligned}
$$

and $\tilde{\eta}=\eta+\eta^{\mathrm{k}} / 3$. Note that, for angular momentum conserving fluids, $\tilde{\eta}=4 \eta / 3$ in $d=3$. Otherwise, the same expressions (17) and (18) are obtained.

In the following, we will need the correlation function $\left\langle\hat{f}_{\alpha}^{R}(\boldsymbol{k}, \omega) \hat{f}_{\beta}^{R}\left(\boldsymbol{k}^{\prime}, \omega^{\prime}\right)\right\rangle$ of the random force. Using the definition of $\boldsymbol{f}^{R}(\boldsymbol{r}, t)$, we find

$$
\begin{aligned}
& \left\langle\hat{f}_{\alpha}^{R}(\boldsymbol{k}, \omega) \hat{f}_{\beta}^{R}\left(\boldsymbol{k}^{\prime}, \omega^{\prime}\right)\right\rangle \\
& =-\sum_{\alpha^{\prime}, \beta^{\prime}} k_{\alpha^{\prime}} k_{\beta^{\prime}}^{\prime}\left\langle\hat{\sigma}_{\alpha \alpha^{\prime}}^{R}(\boldsymbol{k}, \omega) \hat{\sigma}_{\beta \beta^{\prime}}^{R}\left(\boldsymbol{k}^{\prime}, \omega^{\prime}\right)\right\rangle \\
& =\frac{4 \pi k_{B} T}{V} \sum_{\alpha^{\prime}, \beta^{\prime}} k_{\alpha^{\prime}} k_{\beta^{\prime}} \eta_{\alpha \alpha^{\prime} \beta \beta^{\prime}} \delta\left(\omega+\omega^{\prime}\right) \delta_{\boldsymbol{k},-\boldsymbol{k}^{\prime}} .
\end{aligned}
$$

\section{A. Velocity correlation function}

The velocity correlation function $\left\langle\hat{\boldsymbol{v}}(\boldsymbol{k}, \omega) \cdot \hat{\boldsymbol{v}}\left(\boldsymbol{k}^{\prime}, \omega^{\prime}\right)\right\rangle$ in Fourier space can easily be calculated using Eqs. (16)-(19),

$$
\begin{aligned}
\left\langle\hat{\boldsymbol{v}}(\boldsymbol{k}, \omega) \cdot \hat{\boldsymbol{v}}\left(\boldsymbol{k}^{\prime}, \omega^{\prime}\right)\right\rangle= & \frac{4 \pi k_{B} T}{V} k^{2}\left(2 \eta\left|\hat{Q}^{T}\right|^{2}+\tilde{\eta}\left|\hat{Q}^{L}\right|^{2}\right) \\
& \times \delta\left(\omega+\omega^{\prime}\right) \delta_{\boldsymbol{k},-\boldsymbol{k}^{\prime}} .
\end{aligned}
$$

The factor 2 in front of $\left|Q^{T}\right|^{2}$ reflects the two transverse components of vorticity.

The correlation function $\left\langle\boldsymbol{v}(\boldsymbol{k}, t) \cdot \boldsymbol{v}\left(\boldsymbol{k}^{\prime}, 0\right)\right\rangle$ follows by convolution,

$$
\begin{aligned}
\left\langle\boldsymbol{v}(\boldsymbol{k}, t) \cdot \boldsymbol{v}\left(\boldsymbol{k}^{\prime}, 0\right)\right\rangle= & \frac{2 k_{B} T k^{2}}{V} \delta_{\boldsymbol{k},-\boldsymbol{k}^{\prime}} \\
& \times \int\left[2 \eta Q^{T}\left(\boldsymbol{k}, t-t^{\prime}\right) Q^{T}\left(\boldsymbol{k}^{\prime},-t^{\prime}\right)\right. \\
& \left.+\tilde{\eta} Q^{L}\left(\boldsymbol{k}, t-t^{\prime}\right) Q^{L}\left(\boldsymbol{k}^{\prime},-t^{\prime}\right)\right] d t^{\prime} .
\end{aligned}
$$

Fourier transformation yields

$$
Q^{T}(\boldsymbol{k}, t)=\frac{1}{\rho} e^{-\nu k^{2} t} \Theta(t)
$$

for the transverse part, where $\Theta(t)$ is Heaviside's function and $v=\eta / \rho$ denotes the kinematic viscosity. For the longitudinal contribution, we obtain the expression,

$$
\begin{aligned}
Q^{L}(\boldsymbol{k}, t)= & \frac{1}{\rho} e^{-k^{2} \tilde{v} t / 2} \\
& \times\left[\cos (\Omega t)-\sqrt{\frac{k^{2} \tilde{v}^{2}}{4 c^{2}-k^{2} \tilde{v}^{2}}} \sin (\Omega t)\right] \Theta(t)
\end{aligned}
$$

for $4 c^{2} /\left(k^{2} \tilde{v}^{2}\right)>1$, where $\Omega=k^{2} \tilde{v} \sqrt{4 c^{2} /\left(k^{2} \tilde{v}^{2}\right)-1} / 2$, and

$$
\begin{aligned}
Q^{L}(\boldsymbol{k}, t)= & \frac{1}{\rho} e^{-k^{2} \tilde{v} t / 2} \\
& \times\left[\cosh (\Lambda t)-\sqrt{\frac{k^{2} \tilde{v}^{2}}{k^{2} \tilde{v}^{2}-4 c^{2}}} \sinh (\Lambda t)\right] \Theta(t)
\end{aligned}
$$

for $4 c^{2} /\left(k^{2} \tilde{v}^{2}\right)<1$ with $\Lambda=k^{2} \tilde{v} \sqrt{1-4 c^{2} /\left(k^{2} \tilde{v}^{2}\right)} / 2$. 


\section{Transverse velocity correlation function}

With Eq. (20), the transverse velocity correlation function $\left\langle\boldsymbol{v}^{T}(\boldsymbol{k}, t) \cdot \boldsymbol{v}^{T}\left(\boldsymbol{k}^{\prime}, 0\right)\right\rangle$ can be written as

$$
\left\langle\boldsymbol{v}^{T}(\boldsymbol{k}, t) \cdot \boldsymbol{v}^{T}\left(\boldsymbol{k}^{\prime}, 0\right)\right\rangle=\frac{2 k_{B} T \eta}{\pi V} k^{2} \int\left|\hat{Q}^{T}\right|^{2} e^{i \omega t} d \omega \delta_{\boldsymbol{k},-\boldsymbol{k}^{\prime}},
$$

in the stationary state. Evaluation of the integral yields

$$
\left\langle\boldsymbol{v}^{T}(\boldsymbol{k}, t) \cdot \boldsymbol{v}^{T}(-\boldsymbol{k}, 0)\right\rangle=\frac{2 k_{B} T}{\rho V} e^{-v k^{2}|t|} .
$$

The time integral of the normalized correlation function $\left\langle\boldsymbol{v}^{T}(\boldsymbol{k}, t) \cdot \boldsymbol{v}^{T}(-\boldsymbol{k}, 0)\right\rangle /\left\langle\boldsymbol{v}^{T}(\boldsymbol{k}, 0) \cdot \boldsymbol{v}^{T}(-\boldsymbol{k}, 0)\right\rangle$ yields

$$
T(\boldsymbol{k}, t)=\int_{0}^{t} e^{-v k^{2} t^{\prime}} d t^{\prime}=\frac{1}{\nu k^{2}}\left(1-e^{-v k^{2} t}\right) .
$$

Hence, in the limit $t \rightarrow \infty, T(k)$ is proportional to the Oseen tensor $[57,66]$,

$$
\mathbf{O}=\frac{1}{\eta k^{2}}(\mathbf{E}-\mathbf{P})
$$

in $\boldsymbol{k}$ space.

\section{Longitudinal velocity correlation function}

The longitudinal correlation function $\left\langle v^{L}(\boldsymbol{k}, t) v^{L}\left(\boldsymbol{k}^{\prime}, 0\right)\right\rangle$ is most conveniently obtained by the convolution expression in Eq. (21), which yields

$$
\begin{aligned}
& \left\langle v^{L}(\boldsymbol{k}, t) v^{L}(-\boldsymbol{k}, 0)\right\rangle \\
& \quad=\frac{k_{B} T}{\rho V} e^{-\tilde{v} k^{2}|t| / 2}\left[\cos (\Omega|t|)-\sqrt{\frac{k^{2} \tilde{v}^{2}}{4 c^{2}-k^{2} \tilde{v}^{2}}} \sin (\Omega|t|)\right] .
\end{aligned}
$$

For $4 c^{2} /\left(k^{2} \tilde{v}\right)<1$, the hyperbolic functions with the argument $\Lambda$ have to be used as in Eq. (24).

\section{Velocity correlation function in real space}

The velocity correlation function $\left\langle\boldsymbol{v}(\boldsymbol{r}, t) \cdot \boldsymbol{v}\left(\boldsymbol{r}^{\prime}, 0\right)\right\rangle$ of the fluid at a point $\boldsymbol{r}$ at time $t$ and $\boldsymbol{r}^{\prime}$ at $t=0$ follows by Fourier transformation,

$$
\left\langle\boldsymbol{v}(\boldsymbol{r}, t) \cdot \boldsymbol{v}\left(\boldsymbol{r}^{\prime}, 0\right)\right\rangle=\sum_{\boldsymbol{k}}\langle\boldsymbol{v}(\boldsymbol{k}, t) \cdot \boldsymbol{v}(-\boldsymbol{k}, 0)\rangle e^{-i \boldsymbol{k} \cdot\left(\boldsymbol{r}-\boldsymbol{r}^{\prime}\right)},
$$

with $\langle\boldsymbol{v}(\boldsymbol{k}, t) \cdot \boldsymbol{v}(-\boldsymbol{k}, 0)\rangle$ as the sum of the transverse (26) and longitudinal (29) correlation functions. At $t=0$, this expression reduces to

$$
\left\langle\boldsymbol{v}(\boldsymbol{r}, 0) \cdot \boldsymbol{v}\left(\boldsymbol{r}^{\prime}, 0\right)\right\rangle=\frac{3 k_{B} T}{\rho} \delta\left(\boldsymbol{r}-\boldsymbol{r}^{\prime}\right),
$$

which is the equipartition of kinetic energy. Hence, our extension of the thermal stress tensor, with the fluctuations (9), on a nonsymmetric stress tensor satisfies the fluctuationdissipation theorem $[65,67]$.

Adopting the Lagrangian description of the fluid where a fluid element is followed as it moves through space and time, we additionally average the correlation function over the distribution of displacements $\boldsymbol{r}-\boldsymbol{r}^{\prime}$. Hence, Eq. (30) turns into

$$
\langle\boldsymbol{v}(t) \cdot \boldsymbol{v}(0)\rangle=\sum_{\boldsymbol{k}}\langle\boldsymbol{v}(\boldsymbol{k}, t) \cdot \boldsymbol{v}(-\boldsymbol{k}, 0)\rangle\left\langle e^{-i \boldsymbol{k} \cdot\left(\boldsymbol{r}-\boldsymbol{r}^{\prime}\right)}\right\rangle .
$$

Assuming a diffusive motion of the fluid element with Gaussian distributed displacements, we find

$$
\begin{aligned}
& \langle\boldsymbol{v}(t) \cdot \boldsymbol{v}(0)\rangle \\
& \quad=\sum_{\boldsymbol{k}}\langle\boldsymbol{v}(\boldsymbol{k}, t) \cdot \boldsymbol{v}(-\boldsymbol{k}, 0)\rangle \exp \left(-k^{2}\left\langle(\boldsymbol{r}(t)-\boldsymbol{r}(0))^{2}\right\rangle / 6\right) .
\end{aligned}
$$

Here, $\left\langle(\boldsymbol{r}(t)-\boldsymbol{r}(0))^{2}\right\rangle$ indicates the mean square displacement, which, in the simplest case, reduces to $\left\langle(\boldsymbol{r}(t)-\boldsymbol{r}(0))^{2}\right\rangle=6 D t$ with the diffusion coefficient $D$.

In general, the sum over $\boldsymbol{k}$ in Eq. (33) cannot be evaluated analytically. For the transverse velocity correlation function, however, we obtain the expression,

$$
\begin{aligned}
\left\langle\boldsymbol{v}^{T}(t) \cdot \boldsymbol{v}^{T}(0)\right\rangle & =\frac{2 k_{B} T}{\rho(2 \pi)^{3}} \int e^{-v k^{2} t} e^{-D k^{2} t} d^{3} k \\
& =\frac{k_{B} T}{4 \rho} \frac{1}{[\pi(\nu+D) t]^{3 / 2}},
\end{aligned}
$$

in the limit of an infinitely large system $(L \rightarrow \infty)$. Hence, we find the well-known long-time tail of the transverse velocity correlation function [54,68-73].

Examples of the velocity correlation function (33) are displayed in Fig. 1 for an infinitely large and two finite-size systems where

$$
C_{v}(t)=\frac{m}{k_{B} T}\langle\boldsymbol{v}(t) \cdot \boldsymbol{v}(0)\rangle .
$$

MPC characteristic values are chosen for the kinematic viscosity and the diffusion coefficient. As shown in Fig. 1(a) for an infinitely large system, the longitudinal velocity correlation function assumes negative values, whereas, the transverse correlation decays according to the power law of Eq. (34). The sum of both also assumes negative values within a certain time window [Fig. 1(b)] but asymptotically approaches the long-time tail (34) because the sound contribution decays exponentially on that time scale.

The short-time correlation function is determined by contributions from large $\boldsymbol{k}$ values. Hence, we are able to derive an approximate longitudinal velocity correlation function at short times by setting $\Lambda=k^{2} \tilde{v} / 2$ and $\left(1-4 c^{2} /\left(k^{2} \tilde{v}^{2}\right)\right)^{-1 / 2} \approx 1+$ $2 c^{2} /\left(k^{2} \tilde{v}^{2}\right)$. Then, Fourier transformation of the correlation function (29) for an infinite system yields

$$
\begin{aligned}
\left\langle v^{L}(t) v^{L}(0)\right\rangle= & \frac{k_{B} T}{\rho(2 \pi)^{3}} \int e^{-k^{2}(D+\tilde{v} / 2) t}\left[\cosh \left(k^{2} \tilde{v} / 2\right)\right. \\
& \left.-\left(1+\frac{2 c^{2}}{k^{2} \tilde{v}^{2}}\right) \sinh \left(k^{2} \tilde{v} / 2\right)\right] d^{3} k \\
= & \frac{k_{B} T}{8 \rho \pi^{3 / 2}}\left[\frac{1}{[(D+\tilde{v}) t]^{3 / 2}}\right. \\
& \left.-\frac{2 c^{2}}{\tilde{v}^{2}}\left(\frac{1}{\sqrt{D t}}-\frac{1}{\sqrt{(D+\tilde{v}) t}}\right)\right] .
\end{aligned}
$$

This expression gives

$$
t_{0}=\frac{\tilde{v}^{2}}{2 c^{2}(D+\tilde{v})} \frac{\sqrt{D}}{\sqrt{D+\tilde{v}}-\sqrt{D}}
$$

for the time at which the correlation function passes through zero. The comparison with the full expression provides excellent agreement for times $t$ in the vicinity of $t_{0}$ and smaller. 

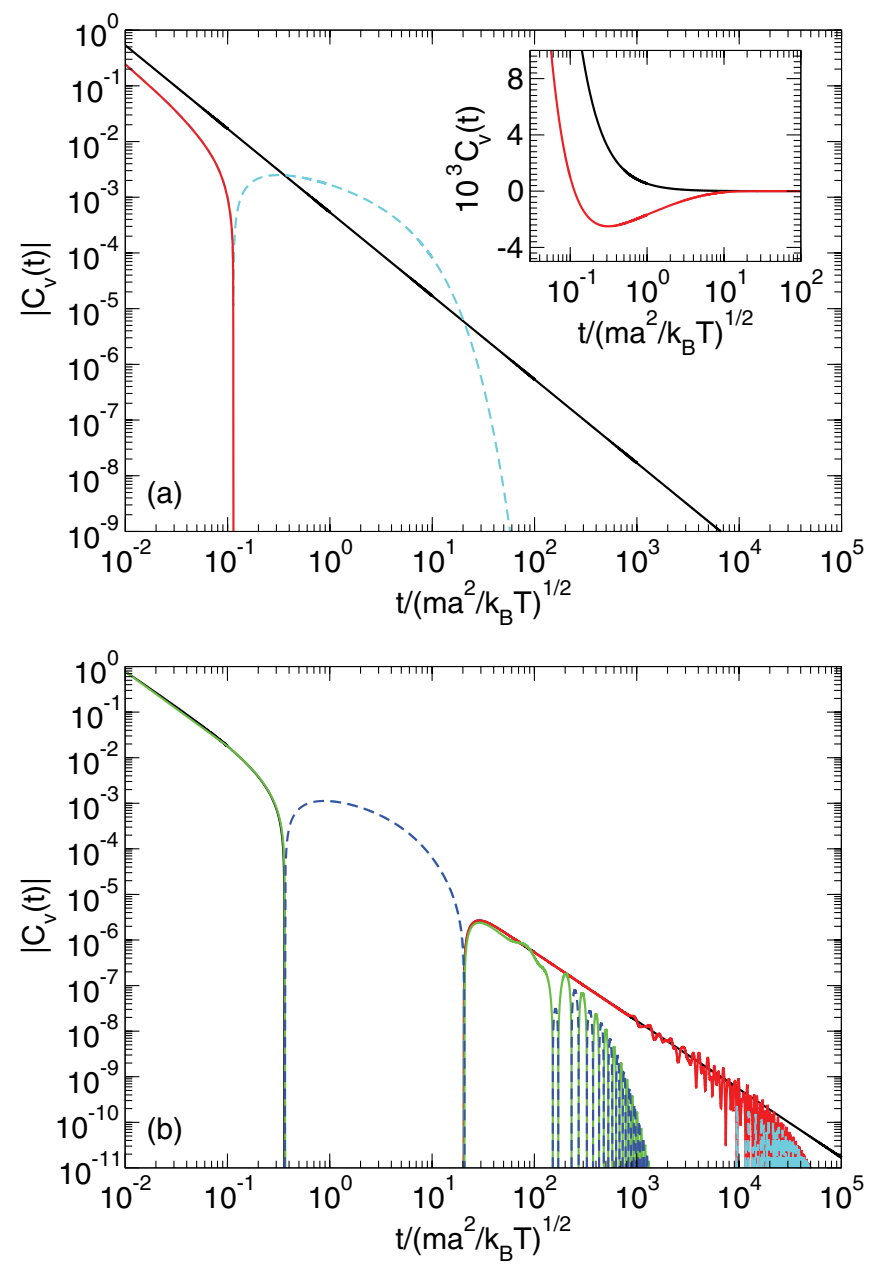

FIG. 1. (Color online) Magnitudes of the velocity autocorrelation functions of infinite and finite size systems for the collision time $h / \sqrt{m a^{2} / k_{B} T}=0.02$. (a) Black, straight line: transverse component of an infinitely large system. The positive longitudinal contribution is displayed as solid curve, red, whereas, the negative one is shown as dashed curve, light blue. The inset shows the same curves with a linear $y$ axis. (b) Black, straight line: Asymptotic correlation function for an infinite system. The other solid lines indicate the positive parts of the correlation functions of finite size systems and the dashed lines their negative parts, with green-blue: $L / a=100$ and red-light blue: 1000. The correlation function for $L / a=100$ deviates early from the asymptotic behavior of an infinite system.

The correlation functions for the finite-size systems of Fig. 1(b) decay exponentially at long times. For such $t$, the correlation function is determined by small $\boldsymbol{k}$ values. For a finite system, the smallest value is $k=2 \pi / L$. Hence, the transverse correlation function decays exponentially as $e^{-t / \tau_{d}}$ with $\tau_{d}=L^{2} /\left[(2 \pi)^{2}(\nu+D)\right]$ for $t>\tau_{d}$, where $\tau_{d} \rightarrow \infty$ for $L \rightarrow \infty$. On shorter time scales $t \ll \tau_{d}$, the curves are hardly distinguishable for the various size systems. The long-time oscillations are caused by the longitudinal velocity correlation function, i.e, by sound. The finite system size leads to a recurrence of sound waves with a period $T=L / c$, where $T$ is the time needed to traverse the simulation box.

\section{MPC SIMULATIONS: RESULTS}

The velocity in Fourier space for a periodic system of discrete particles is

$$
\boldsymbol{v}(\boldsymbol{k}, t)=\frac{1}{N} \sum_{i=1}^{N} \boldsymbol{v}_{i}(t) e^{i \boldsymbol{k} \cdot \boldsymbol{r}_{i}(t)},
$$

with the $\boldsymbol{k}$ values given after Eq. (14). With these velocities, we can calculate the various correlation functions discussed in Sec. III and can compare them to those of the Stokes equation.

\section{A. Transverse velocity correlation function}

Normalized transverse velocity correlation functions,

$$
C_{v}^{T}(t)=\left\langle\boldsymbol{v}^{T}(\boldsymbol{k}, t) \cdot \boldsymbol{v}^{T}(-\boldsymbol{k}, 0)\right\rangle /\left\langle\boldsymbol{v}^{T}(\boldsymbol{k}, 0) \cdot \boldsymbol{v}^{T}(-\boldsymbol{k}, 0)\right\rangle
$$

are displayed in Fig. 2. The correlation functions decay exponentially, exactly as predicted by Eq. (26). This has been shown before in Ref. [51] for an isoenergetic system.

To analyze the length-scale dependence of hydrodynamics in a MPC fluid, we calculate the time integral of the velocity correlation function [cf. Eq. (27)]. The results for various $k$ values are presented in Fig. 3. The simulation data agree very well with the theoretical prediction over the considered range of $k$ values and for the whole time scale. Even the short-time behavior at a few MPC collisions only is in excellent agreement with the theoretical expression. The various curves reach a plateau value for time scales $t>\tau_{k}=\left(v k^{2}\right)^{-1}$, which depends on the particular length scale. The plateau is reached earlier at smaller length scales, i.e, large $k$ values.

As pointed out in Sec. III A1, the plateau values $T(\boldsymbol{k})=$ $\lim _{t \rightarrow \infty} T(\boldsymbol{k}, t)$ are related to the Oseen tensor (28). Hence, we are able to probe the length-scale dependence of hydrodynamics in a MPC fluid, i.e., its agreement with the Oseen prediction. A similar study has been performed in Ref. [74] for a Lennard-Jones fluid (see also Ref. [75]).

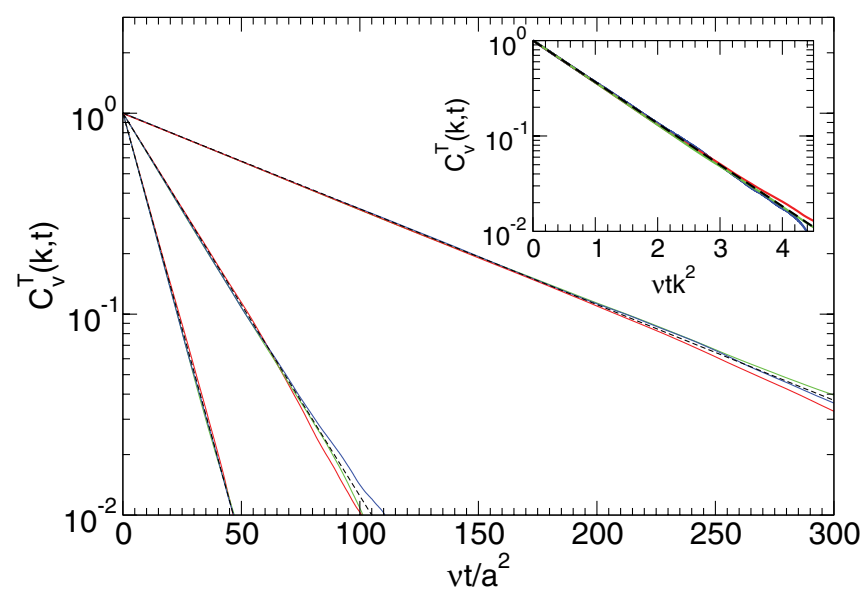

FIG. 2. (Color online) From right to left: transverse velocity autocorrelation functions of a MPC fluid for $k=2 \pi n / L$ with $n=1,2,3$ and $L / a=60$. The collision time steps are blue, dark gray: $h / \sqrt{m a^{2} /\left(k_{B} T\right)}=0.5$; green, light gray: 0.1 ; and red: 0.01 . The individual curves are hardly distinguishable. The corresponding theoretical prediction (26) is represented by a dashed line. Inset: universal dependence on $v k^{2} t$. 


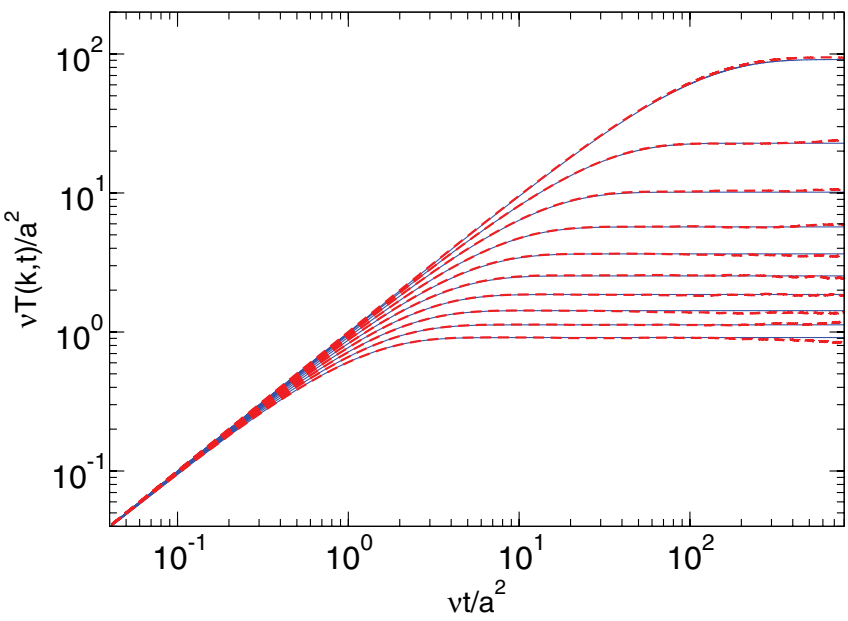

FIG. 3. (Color online) Time dependence of the integrated transverse velocity correlation function (39). The collision time is $h / \sqrt{m a^{2} /\left(k_{B} T\right)}=0.01$ and $L / a=60$. From top to bottom, the dashed lines correspond to the $k$ values: $k=2 \pi n / L, n=1, \ldots, 10$. The solid lines indicate the theoretical expression (27).

Figure 4 depicts the $k$ dependence of $T(\boldsymbol{k})$ for various collision time steps $h$. For sufficiently small $k$ values, $T(\boldsymbol{k})$ follows the prediction of the Stokes equation and, hence, shows the same dependence as the Oseen tensor. Above a certain value, which depends on the collision time step, $T(\boldsymbol{k})$ itself approaches a plateau. Hence, below a certain length scale, no hydrodynamic interactions are present anymore.

Applying the molecular chaos assumption, the asymptotic behavior is calculated in the Appendix. As shown in Fig. 4, the theoretical expression captures the small scale behavior.

A characteristic length scale $\lambda_{c}$, separating the hydrodynamic from the nonhydrodynamic regime, is obtained by the intercept of the Oseen type dependence $T(\boldsymbol{k})=1 /\left(\nu k^{2}\right)$

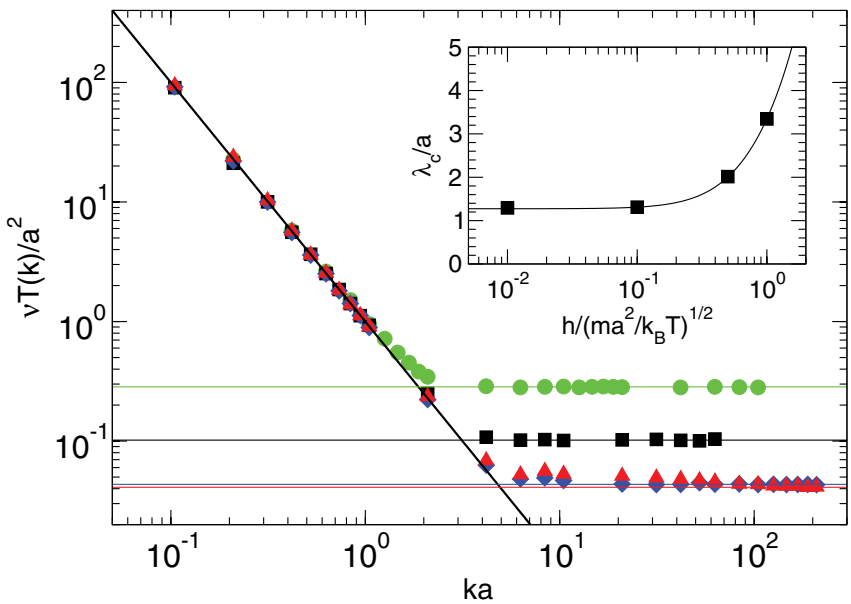

FIG. 4. (Color online) Dependence of $T(k)=\lim _{t \rightarrow \infty} T(k, t)$ (27) on the wave number for the collision times $\boldsymbol{\Delta}: h / \sqrt{m a^{2} /\left(k_{B} T\right)}=$ 0.01 ; $: 0.1$; $\mathbf{\square}: 0.5$; and $\bullet: 1.0$. The thick solid line indicates the dependence $1 / k^{2}$, corresponding to the Oseen tensor. The horizontal lines are the theoretical predictions for the plateau values of $v T(k)$ [Eq. (A5)]. The inset shows the theoretical prediction for the characteristic length scale $\lambda_{c}$; solid line: Eq. (40) and squares: values extracted from the simulations. with the asymptotic dependence $T(\boldsymbol{k})=T_{\mathrm{mc}}(\boldsymbol{k})=h / 2$, which yields

$$
\lambda_{c}=\pi \sqrt{2 \nu h} .
$$

The viscosity of a MPC fluid is dominated by the collisional part with its dependence $\eta^{\mathrm{c}} \sim h^{-1}$ on collision time in the fluid regime $h \rightarrow 0[2-5,50,59,76,77]$. Hence, we obtain the asymptotic minimal characteristic length,

$$
\lambda_{c}=\pi a \sqrt{\frac{\gamma}{6}},
$$

with $\gamma=2(1-\cos \alpha)\left(1-1 /\left\langle N_{c}\right\rangle\right) / 3$ [see Eq. (A2)]. This yields $\lambda_{c} \approx 1.3 a$ for the parameters of Sec. II, consistent with the expectation that there is no hydrodynamics on length scales below the collision cell size. In the opposite limit of large collision times $h / \sqrt{m a^{2} /\left(k_{B} T\right)}>1$, viscosity is dominated by $\eta^{\mathrm{k}}$, which increases linearly with the collision time. Hence, $\lambda_{c} \sim h$ in that regime and increases with the collision time. With the parameters of Sec. II, we find $\lambda_{c} \approx$ $3.1 a h \sqrt{k_{B} T /\left(m a^{2}\right)}$.

The dependence of $\lambda_{c}$ on the collision time step is presented in the inset of Fig. 4. The simulation data are obtained by the intercept of the function $T(\boldsymbol{k})=1 /\left(v k^{2}\right)$ with constants fitted to the simulation data for the various $h$ values and for $k a>10$. Evidently, the theoretical expression describes the experimental data very well.

\section{B. Longitudinal velocity correlation function}

Results for the longitudinal velocity correlation function,

$$
C_{v}^{L}(\boldsymbol{k}, t)=\left\langle v^{L}(\boldsymbol{k}, t) v^{L}(-\boldsymbol{k}, 0)\right\rangle /\left\langle v^{L}(\boldsymbol{k}, 0) v^{L}(-\boldsymbol{k}, 0)\right\rangle
$$

are presented in Fig. 5. The simulation data agree well with the theoretical prediction (29) for collision time steps $h / \sqrt{m a^{2} /\left(k_{B} T\right)} \ll 1$. Since $\eta^{\mathrm{k}} \ll \eta$ for these collision times,

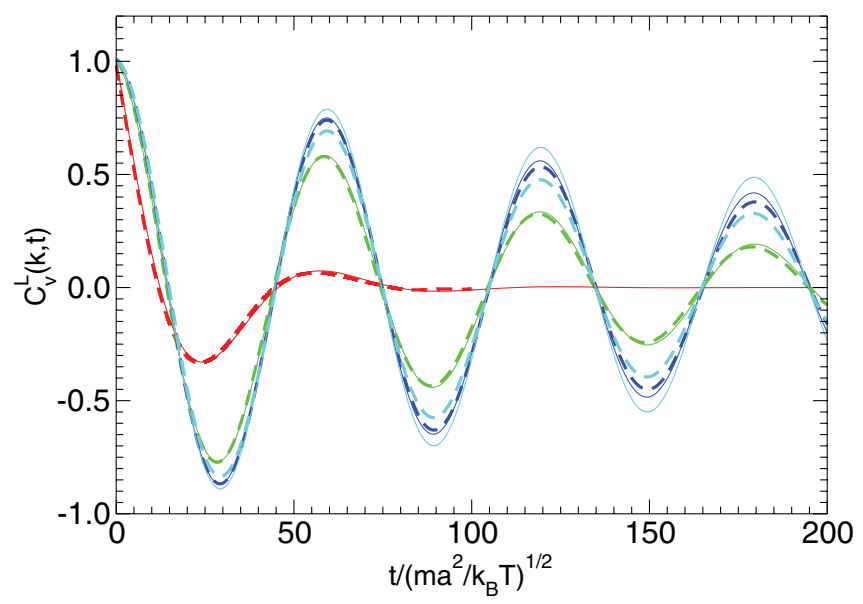

FIG. 5. (Color online) Longitudinal velocity autocorrelation functions for the collision times red: $h / \sqrt{m a^{2} /\left(k_{B} T\right)}=0.01$; green: 0.05 ; blue: 0.1 ; and light blue: $1.0 ; k=2 \pi / L$, and $L / a=60$. The thin solid lines are theoretical predictions according to Eq. (29). Solid lines: the order of the maxima at $t / \sqrt{m a^{2} /\left(k_{B} T\right)} \approx 60$ corresponds to bottom to top: $h / \sqrt{m a^{2} /\left(k_{B} T\right)}=0.01,0.05,0.1$, and 1.0. 
$\tilde{\eta} \approx \eta$, and the decay of the longitudinal velocity correlation function is governed by the collisional contribution of the viscosity. Yet, $\tilde{\eta} \approx \eta$ reflects the nonsymmetry of the collisional stress tensor (7), otherwise, $\tilde{\eta}=4 \eta / 3$ [60].

We observe some deviations between the simulation results and the theoretical prediction for $h / \sqrt{m a^{2} /\left(k_{B} T\right)} \gtrsim 1$ as shown in Fig. 5. To understand the origin and consequence of the obtained deviations at large $h$, we recall that the theoretical calculations of Sec. III are based on an isothermal system. This implies a decoupling of the longitudinal momentum (velocity) current fluctuations from that of the (longitudinal) energy current fluctuations [78,79]. Note that the transverse momentum current fluctuations are always decoupled. The decoupling assumption seems to break down for large collision time steps, although we employ a local thermostat. As long as the mean-free path of a MPC particle-it is equal to $h c$-is smaller than a collision cell, momentum transfer is governed by collisional interactions. For larger mean-free paths, momentum and energy are also transported in the streaming step. Hence, the system is not (locally) isothermal anymore. To fully describe the transport properties in this case, the coupling of the momentum and energy current has to be taken into account. The modifications of the MPC fluid-density fluctuations have been studied in Ref. [80] as a function of the thermalization interval. The authors find an increasing influence of energy transport on the correlation function with an increasing interval between the scaling of the velocities. We would like to emphasize that density correlations for an adiabatic MPC fluid have been studied in Ref. [51]. Since we typically apply collision times $h / \sqrt{m a^{2} /\left(k_{B} T\right)}<0.1$, the provided description applies.

As is well known and is confirmed by our simulations, the time integral $\int_{0}^{\infty} C_{v}^{L} d t$ vanishes, and the longitudinal mode does not contribute to the fluid self-diffusion coefficient.

Finally, Fig. 6 shows the spectrum of the longitudinal velocity autocorrelation function (20). Again, the simulation

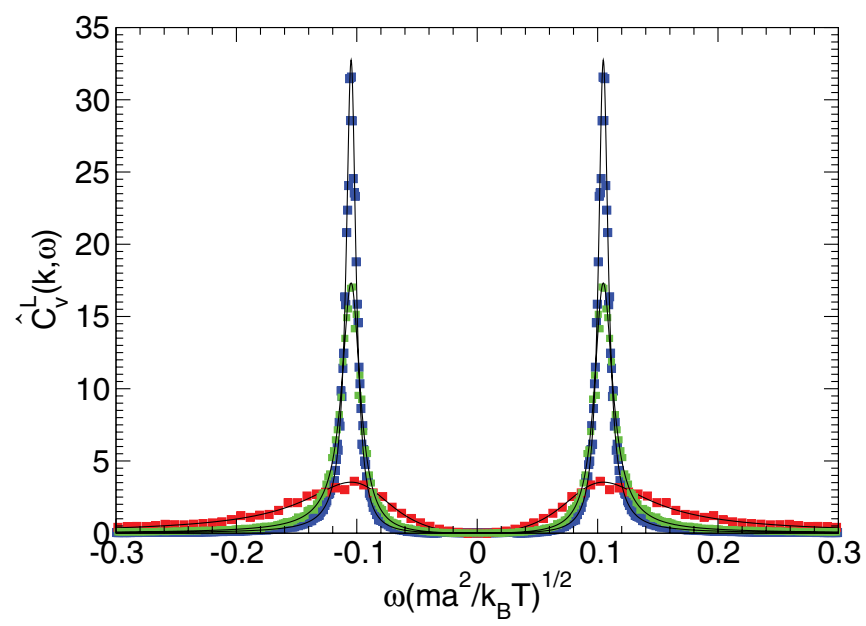

FIG. 6. (Color online) Spectra $\hat{C}_{v}^{L}(\boldsymbol{k}, \omega)$ of the longitudinal velocity autocorrelation function (42) for the collision times red, lower: $h / \sqrt{m a^{2} /\left(k_{B} T\right)}=0.01$; green, middle: 0.05; and blue, upper: 0.1 , and $k=2 \pi /(60 a)$. The lines are obtained from the theoretical expression (20). results are well described by the theoretical approach. In particular, the frequencies at the peak positions $\omega_{c}=c k$ confirm that our systems are isothermal for the considered collision times.

We also calculated the dynamic structure factor $[58,78]$ of the MPC fluid. As expected for an isothermal system, there is no Rayleigh line at $\omega=0$ but only two Brillouin lines at $\omega_{c}= \pm c k[51,78,80]$. Hence, energy (or heat) diffusion is suppressed by the applied thermostat.

The quantitative agreement between the theoretical and the simulation results confirms that the bulk viscosity is either zero or negligibly small for the considered MPC fluid with $h / \sqrt{m a^{2} /\left(k_{B} T\right)} \lesssim 0.1$ since a non-negligible bulk viscosity would affect the longitudinal velocity correlation function [60].
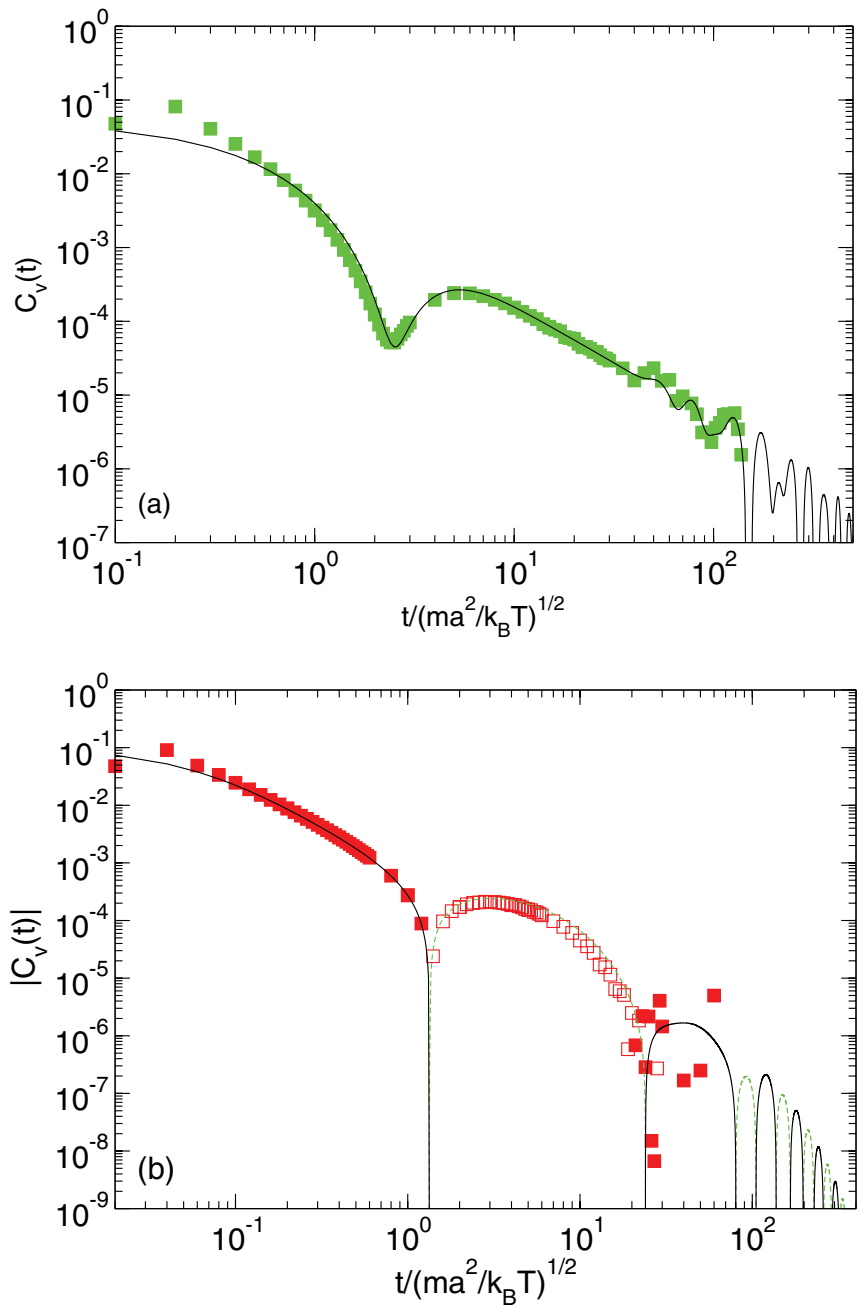

FIG. 7. (Color online) Symbols: magnitude of the velocity autocorrelation function (35) of a MPC fluid for the collision times (a) $h / \sqrt{m a^{2} /\left(k_{B} T\right)}=0.1$ and (b) 0.02 . Filled symbols indicate positive correlation functions, and open symbols indicate negative correlation functions. Solid lines: the theoretical results are obtained from Eq. (33) with Eqs. (26) and (29) where solid lines indicate positive correlation functions, and dashed lines indicate negative ones. 


\section{Velocity correlation function in real space}

Velocity correlation functions of a MPC fluid in real space are presented in Fig. 7 for two collision time steps. The simulation data are well described by the theoretical expression Eq. (33) with Eqs. (26) and (29) over several decades in time. We would like to emphasize that we include the full mean square displacement of a MPC particle (33) and not simply the linear dependence on time. The latter yields a slightly different theoretical curve, in particular, in the vicinity of the minimum. The theoretical approach even reproduces the oscillations due to sound at long times. However, we have to introduce an upper cutoff for the $k$ values. As discussed in Sec. IV A, the hydrodynamic description of the MPC fluid breaks down below a certain length scale. To achieve a good fit over a long time range, the maximum $k$ value is $k_{n}=2 \pi n / 60$ with $n=16$ for $h / \sqrt{m a^{2} /\left(k_{B} T\right)}=0.1$ and $n=21$ for 0.02 , respectively. This corresponds to the lower length scales $\approx 3.8 a$ and $\approx 2.9 a$, respectively. Both values are somewhat above the theoretically estimated critical length scale $\lambda_{c} \approx a$.

The deviation between the theoretical expression and the simulation results at short times is also related to the cutoff in $k$ values. As noted before, the expression for the theoretical correlation function is determined by large $k$ values at short times. Here, however, the theoretical and simulation results deviate because the MPC solvent does not exhibit hydrodynamic behavior anymore for $2 \pi / \lambda_{c}<k<\infty$.

The derivation of a theoretical correlation function, which matches the simulation data over the whole considered time window, is a challenge. Such an endeavor requires the knowledge of the correlation functions $C_{v}^{T}(\boldsymbol{k}, t)$ and $C_{v}^{L}(\boldsymbol{k}, t)$ in the crossover regime between full hydrodynamics and no hydrodynamics. We expect the adopted linearized hydrodynamic description of the MPC fluid to fail already on length scales somewhat larger than $\lambda_{c}$ for the longitudinal mode. The discrete character of the particle system and the (local) fluctuations in particle number can only approximately be captured by the wave equation adopted to derive Eq. (12).

\section{CONCLUSIONS}

We have studied the hydrodynamic properties of a MPC fluid. We determined the transverse and longitudinal velocity correlation functions in Fourier space and calculated the velocity correlation function in real space for various collision times. In particular, we investigated the validity range of a hydrodynamic tensor description in terms of the Oseen tensor, i.e., the spatial scale over which the velocity correlation function exhibits the dependence $\sim k^{-2}$. We find that this relation is violated on length scales less than $\lambda_{c}=\pi \sqrt{2 \nu h}$ (41). The characteristic value $\lambda_{c}$ is independent of the collision time for $h / \sqrt{m a^{2} /\left(k_{B} T\right)} \ll 1$ where it is comparable with a collision cell. For large $h$ values, $\lambda_{c}$ increases linearly with $h$. Hence, a fluidlike MPC system, where $h / \sqrt{m a^{2} /\left(k_{B} T\right)} \lesssim 1$, displays hydrodynamic behavior down to the collision cell level in agreement with other simulation papers, such as a rotating colloid near a solid wall [11].

Moreover, we analyzed the system-size dependence of the velocity autocorrelation function. We demonstrated that the correlation function decays exponentially at long times for such systems because the correlation function is determined by the smallest $k$ value. In addition, we addressed the role of sound in an isothermal system on the velocity correlation function. We find that sound implies negative correlation functions [15], which are more pronounced for smaller collision time steps. At long times, when sound waves traverse the periodic simulation box, oscillations appear in the autocorrelation function. These oscillations complicate the identification of a long-time tail, displayed by the correlation function.

For an analytical description of the MPC fluid, we chose a fluctuating hydrodynamics approach based on the linearized Landau-Lifshitz Navier-Stokes equation. Since the stress tensor of the SRD implementation of a MPC fluid, where fluid velocities are rotated, is nonsymmetric, we extended the fluid stress-tensor correlation functions to satisfy the fluctuationdissipation theorem. The comparison of the theoretical results for various fluid velocity correlations with simulations yields excellent agreement over a wide range of length and time scales. It is the analytical description which provides the necessary insight into the characteristics of the correlation functions to understand and to quantify the simulation findings.

\section{ACKNOWLEDGMENTS}

Helpful discussions with T. Ihle, S. Messlinger, H. Noguchi, G. Sutmann, and U. D. Schiller are gratefully acknowledged. Financial support by the German Research Foundation (DFG) within SFB TR6 and the European Union through FP7-Infrastructures ESMI (Grant No. 262348) is gratefully acknowledged. We are grateful to the Jülich Supercomputer Centre (JSC) for allocation of a CPU-time grant.

\section{APPENDIX: MPC VELOCITY CORRELATION FUNCTION: MOLECULAR CHAOS APPROXIMATION}

Applying the molecular chaos approximation, i.e, there are no correlations between different MPC particles, the velocity correlation function of a MPC particles is [81]

$$
\left\langle\boldsymbol{v}_{i}(t) \cdot \boldsymbol{v}_{i}(0)\right\rangle=\left\langle\boldsymbol{v}_{i}^{2}(0)\right\rangle(1-\gamma)^{n},
$$

with $t=n h$,

$$
\gamma=\frac{2}{3}(1-\cos \alpha)\left(1-\frac{1}{\left\langle N_{c}\right\rangle}\right),
$$

and the MPC parameters defined in Sec. II. Under this assumption, there is no difference between transverse and longitudinal velocity correlation functions anymore. In Fourier space (38), the correlation becomes

$$
\langle\boldsymbol{v}(\boldsymbol{k}, t) \cdot \boldsymbol{v}(-\boldsymbol{k}, 0)\rangle=\frac{3 k_{B} T}{m N}(1-\gamma)^{n} e^{-n h D k^{2}}
$$

[cf. Eqs. (32) and (33)]. For the discrete-time random process, the expression (27) is replaced by

$$
T_{\mathrm{mc}}(\boldsymbol{k})=h \sum_{n=0}^{\infty} \frac{\langle\boldsymbol{v}(\boldsymbol{k}, n h) \cdot \boldsymbol{v}(-\boldsymbol{k}, 0)\rangle}{\langle\boldsymbol{v}(\boldsymbol{k}, 0) \cdot \boldsymbol{v}(-\boldsymbol{k}, 0)\rangle}-\frac{h}{2},
$$

in the limit $t \rightarrow \infty$. Since $\left[(1-\gamma) e^{-h D k^{2}}\right]^{n}<1$, we find

$$
T_{\mathrm{mc}}(\boldsymbol{k})=h\left(\frac{1}{1-(1-\gamma) e^{-h D k^{2}}}-\frac{1}{2}\right) .
$$

This expression reduces to $T_{\mathrm{mc}}(\boldsymbol{k})=h / 2$ in the limit $\boldsymbol{k} \rightarrow \infty$. 
[1] A. Malevanets and R. Kapral, J. Chem. Phys. 110, 8605 (1999).

[2] A. Malevanets and R. Kapral, J. Chem. Phys. 112, 7260 (2000).

[3] R. Kapral, Adv. Chem. Phys. 140, 89 (2008).

[4] G. Gompper, T. Ihle, D. M. Kroll, and R. G. Winkler, Adv. Polym. Sci. 221, 1 (2009).

[5] T. Ihle and D. M. Kroll, Phys. Rev. E 63, 020201(R) (2001).

[6] T. Ihle and D. M. Kroll, Phys. Rev. E 67, 066706 (2003).

[7] E. Allahyarov and G. Gompper, Phys. Rev. E 66, 036702 (2002).

[8] S. H. Lee and R. Kapral, J. Chem. Phys. 121, 11163 (2004).

[9] M. Hecht, J. Harting, T. Ihle, and H. J. Herrmann, Phys. Rev. E 72, 011408 (2005).

[10] J. T. Padding and A. A. Louis, Phys. Rev. E 74, 031402 (2006).

[11] I. O. Götze, H. Noguchi, and G. Gompper, Phys. Rev. E 76, 046705 (2007).

[12] M. K. Petersen, J. B. Lechman, S. J. Plimpton, G. S. Grest, P. J. in 't Veld, and P. R. Schunk, J. Chem. Phys. 132, 174106 (2010).

[13] J. K. Whitmer and E. Luijten, J. Phys.: Condens. Matter 22, 104106 (2010).

[14] T. Franosch, M. Grimm, M. Belushkin, F. M. Mor, G. Foffi, L. Forró, and S. Jeney, Nature (London) 478, 85 (2011).

[15] M. Belushkin, R. G. Winkler, and G. Foffi, J. Phys. Chem. B 115, 14263 (2011).

[16] A. Malevanets and J. M. Yeomans, Europhys. Lett. 52, 231 (2000).

[17] M. Ripoll, K. Mussawisade, R. G. Winkler, and G. Gompper, Europhys. Lett. 68, 106 (2004).

[18] K. Mussawisade, M. Ripoll, R. G. Winkler, and G. Gompper, J. Chem. Phys. 123, 144905 (2005).

[19] C.-C. Huang, R. G. Winkler, G. Sutmann, and G. Gompper, Macromolecules 43, 10107 (2010).

[20] A. Lamura, G. Gompper, T. Ihle, and D. M. Kroll, Europhys. Lett. 56, 319 (2001).

[21] R. G. Winkler, K. Mussawisade, M. Ripoll, and G. Gompper, J. Phys.: Condens. Matter 16, S3941 (2004).

[22] J. T. Padding and A. A. Louis, Phys. Rev. Lett. 93, 220601 (2004).

[23] M. Ripoll, P. Holmqvist, R. G. Winkler, G. Gompper, J. K. G. Dhont, and M. P. Lettinga, Phys. Rev. Lett. 101, 168302 (2008).

[24] A. Wysocki, C. P. Royall, R. G. Winkler, G. Gompper, H. Tanaka, A. van Blaaderen, and H. Löwen, Soft Matter 5, 1340 (2009).

[25] I. O. Götze and G. Gompper, Europhys. Lett. 92, 64003 (2010).

[26] S. P. Singh, R. G. Winkler, and G. Gompper, Phys. Rev. Lett. 107, 158301 (2011).

[27] M. A. Webster and J. M. Yeomans, J. Chem. Phys. 122, 164903 (2005).

[28] J. F. Ryder and J. M. Yeomans, J. Chem. Phys. 125, 194906 (2006).

[29] M. Ripoll, R. G. Winkler, and G. Gompper, Phys. Rev. Lett. 96, 188302 (2006).

[30] L. Cannavacciuolo, R. G. Winkler, and G. Gompper, Europhys. Lett. 83, 34007 (2008).

[31] S. Frank and R. G. Winkler, Europhys. Lett. 83, 38004 (2008).

[32] R. Chelakkot, R. G. Winkler, and G. Gompper, Europhys. Lett. 91, 14001 (2010).

[33] A. Nikoubashman and C. N. Likos, J. Chem. Phys. 133, 074901 (2010).

[34] D. A. Fedosov, S. P. Singh, A. Chatterji, R. G. Winkler, and G. Gompper, Soft Matter 8, 4109 (2012).

[35] H. Noguchi and G. Gompper, Phys. Rev. Lett. 93, 258102 (2004).
[36] H. Noguchi and G. Gompper, Proc. Natl. Acad. Sci. USA 102, 14159 (2005).

[37] J. L. Mcwhirter, H. Noguchi, and G. Gompper, Proc. Natl. Acad. Sci. USA 106, 6039 (2009).

[38] S. Ji, R. Jiang, R. G. Winkler, and G. Gompper, J. Chem. Phys. 135, 134116 (2011).

[39] G. Rückner and R. Kapral, Phys. Rev. Lett. 98, 150603 (2007).

[40] I. O. Götze and G. Gompper, Phys. Rev. E 82, 041921 (2010).

[41] M. Yang and M. Ripoll, Phys. Rev. E 84, 061401 (2011).

[42] J. Elgeti and G. Gompper, Europhys. Lett. 85, 38002 (2009).

[43] D. J. Earl, C. M. Pooley, J. F. Ryder, I. Bredberg, and J. M. Yeomans, J. Chem. Phys. 126, 064703 (2007).

[44] J. Elgeti, U. B. Kaupp, and G. Gompper, Biophys. J. 99, 1018 (2010).

[45] S. Y. Reigh, R. G. Winkler, and G. Gompper, Soft Matter 8, 4363 (2012).

[46] T. Ihle, E. Tüzel, and D. M. Kroll, Europhys. Lett. 73, 664 (2006).

[47] E. Tüzel, G. Pan, T. Ihle, and D. M. Kroll, Europhys. Lett. 80, 40010 (2007).

[48] G. Sutmann, C.-C. Huang, R. G. Winkler, and G. Gompper, in John von Neumann Institute for Computing NIC Symposium 2010, edited by G. Münster, D. Wolf, and M. Kremer, IAS Series, Vol. 3 (Forschungszentrum Jülich, Jülich, 2010), pp. 287-294.

[49] C. M. Pooley and J. M. Yeomans, J. Phys. Chem. B 109, 6505 (2005).

[50] T. Ihle, E. Tüzel, and D. M. Kroll, Phys. Rev. E 72, 046707 (2005).

[51] E. Tüzel, T. Ihle, and D. M. Kroll, Phys. Rev. E 74, 056702 (2006).

[52] T. Ihle, Phys. Chem. Chem. Phys. 11, 9667 (2009).

[53] H. Noguchi, N. Kikuchi, and G. Gompper, Europhys. Lett. 78, 10005 (2007).

[54] B. U. Felderhof, J. Chem. Phys. 123, 044902 (2005).

[55] J. X. Zhu, D. J. Durian, J. Muller, D. A. Weitz, and D. J. Pine, Phys. Rev. Lett. 68, 2559 (1992).

[56] S. Henderson, S. Mitchell, and P. Bartlett, Phys. Rev. Lett. 88, 088302 (2002).

[57] M. Doi and S. F. Edwards, The Theory of Polymer Dynamics (Clarendon, Oxford, 1986).

[58] C.-C. Huang, A. Chatterji, G. Sutmann, G. Gompper, and R. G. Winkler, J. Comput. Phys. 229, 168 (2010).

[59] R. G. Winkler and C.-C. Huang, J. Chem. Phys. 130, 074907 (2009).

[60] L. D. Landau and E. M. Lifshitz, Fluid Mechanics (Pergamon, London, 1960).

[61] D. Bedeaux and P. Mazur, Physica 76, 247 (1974).

[62] J. Bonet Avalos, J. M. Rubí, and D. Bedeaux, Macromolecules 24, 5997 (1991).

[63] M. M. Denn, Process Fluid Mechanics (Prentice Hall, Englewood Cliffs, NJ, 1980).

[64] R. B. Bird, R. C. Armstrong, and O. Hassager, Dynamics of Polymer Liquids, Vol. 1 (Wiley, New York, 1987).

[65] U. D. Schiller, Doctoral thesis, Johannes Gutenberg-Universität, Mainz, 2008.

[66] J. K. G. Dhont, An Introduction to Dynamics of Colloids (Elsevier, Amsterdam, 1996).

[67] H. Risken, The Fokker-Planck Equation (Springer, Berlin, 1989).

[68] B. J. Alder and T. E. Wainwright, Phys. Rev. A 1, 18 (1970). 
[69] R. Zwanzig and M. Bixon, Phys. Rev. A 2, 2005 (1970).

[70] M. H. Ernst, E. H. Hauge, and J. M. J. van Leeuwen, Phys. Rev. A 4, 2055 (1971).

[71] E. H. Hauge and A. Martin-Löf, J. Stat. Phys. 7, 259 (1973).

[72] E. J. Hinch, J. Fluid Mech. 72, 499 (1975).

[73] G. L. Paul and P. N. Pusey, J. Phys. A 14, 3301 (1981).

[74] B. Dünweg and K. Kremer, J. Chem. Phys. 99, 6983 (1993).

[75] P.-G. de Gennes, Scaling Concepts in Polymer Physics (Cornell University Press, Ithaca, NY, 1979).
[76] N. Kikuchi, C. M. Pooley, J. F. Ryder, and J. M. Yeomans, J. Chem. Phys. 119, 6388 (2003).

[77] H. Noguchi and G. Gompper, Phys. Rev. E 78, 016706 (2008).

[78] J.-P. Hansen and I. R. McDonald, Theory of Simple Liquids (Academic, London, 1986).

[79] J. P. Boon and S. Yip, Molecular Hydrodynamics (Dover, New York, 1980).

[80] H. Híjar and G. Sutmann, Phys. Rev. E 83, 046708 (2011).

[81] M. Ripoll, K. Mussawisade, R. G. Winkler, and G. Gompper, Phys. Rev. E 72, 016701 (2005). 